\title{
Aldohexose Malabsorption in Preterm Pigs Is Directly Related to the Severity of Necrotizing Enterocolitis
}

\author{
RANDAL K. BUDDINGTON, STINE B. BERING, THOMAS THYMANN, AND PER T. SANGILD
}

Department of Health and Sports Sciences [R.K.B.], University of Memphis, Memphis, Tennessee 39152; Department of Human Nutrition [S.B.B., T.T., P.T.S.], University of Copenhagen, DK-1958 Frederiksberg C, Denmark

\begin{abstract}
Necrotizing enterocolitis (NEC) causes morbidity and mortality among preterm infants and is associated with nutrient malabsorption. Therefore, a preterm pig model that spontaneously develops NEC was used to investigate the relationship between severity of NEC lesions and galactose absorption in vivo and carriermediated glucose absorption by intact mid small intestine. Preterm pigs collected by caesarian section at $92 \%$ of gestation received parenteral nutrition with and without minimal enteral nutrition for $48 \mathrm{~h}$ before conversion to enteral nutrition with colostrum or an enteral formula. Pigs were killed when symptoms of NEC were observed or after 36-40 h of enteral nutrition. NEC lesions decreased in vivo absorption of galactose and mannitol by more than $50 \%$ and abolished carrier-mediated glucose uptake by tissues with lesions. Moreover, when NEC lesions were restricted to the colon, small intestinal tissues that seemed clinically healthy had decreased in vitro glucose absorption due to reduced uptake via the sodiumdependent glucose transporter with little or no involvement of the apical facilitative glucose carrier. The present findings reveal a direct relationship between the severity of NEC lesions and the magnitude of sugar malabsorption that is detectable before clinical symptoms are evident. (Pediatr Res 63: 382-387, 2008)
\end{abstract}

$\mathrm{N}$ ecrotizing enterocolitis (NEC) afflicts up to $28 \%$ of premature infants (1). Although the risk of NEC may or may not be declining $(2,3)$, the incidence of NEC will rise due to increases in the numbers and survival rates of preterm infants $(4,5)$. The etiology of NEC is multifactorial with prematurity and bacteria as consistent factors (5), with formula instead of breast milk also implicated $(6,7)$.

Preterm infants at risk of NEC have underdeveloped capacities to absorb monosaccharides, corresponding with food intolerance and increased breath hydrogen excretion $(8,9)$, with the magnitude of malabsorption related to degree of prematurity (10). Apparently, unabsorbed dietary nutrients cause overgrowth of bacteria in the gastrointestinal tract (GIT) and the resulting higher concentrations of short chain fatty acids damage the intestine and initiate NEC (11).

Bowel resection is a common intervention for infants with NEC unresponsive to treatment or with bowel perforation $(1,12)$, and the resulting short-bowel syndrome further compromises nutrient absorption (13). Of clinical importance is

Received October 17, 2007; accepted December 16, 2007

Correspondence: Randal K. Buddington, Ph.D., Department of Health and Sport Sciences, 311 Elma Roane Fieldhouse, University of Memphis, Memphis, TN 39152; e-mail: rbddngtn@memphis.edu

Funding for the research was provided by The Danish Research Councils.

DOI: 10.1203/PDR.0b013e318165bfed understanding if NEC lesions in one GIT region affect absorptive capacities of the entire small intestine, exacerbating the malabsorption and compromising recovery from bowel resection. Therefore, the present study used a preterm pig model (14) to evaluate the impact of NEC on nutrient absorption and particularly if absorption by unafflicted regions is compromised by NEC lesions in other GIT regions. The findings improve our understanding of the impact of NEC on small intestine absorptive functions and will facilitate development of interventions for preventing and treating NEC.

\section{METHODS}

The research used 65 premature pigs obtained by caesarian section (15) at $92 \%$ of gestation from four sows (Danish Landrace $\times$ Large White) and had been approved by National Committee on Animal Experimentation, Denmark. NEC was induced following established methods (14), except for the use of a 48-h postnatal period of parenteral nutrition (PN) to increase the incidence and severity of NEC (unpublished data) and improve clinical relevance, as many preterm infants do not tolerate enteral feeding for $1-2 \mathrm{~d}$ after delivery.

For the first $12 \mathrm{~h}$ after delivery, PN (16) was provided via a vascular catheter at $4 \mathrm{~mL} / \mathrm{kg} / \mathrm{h}$ after which the rate was increased to $6-8 \mathrm{~mL} / \mathrm{kg} / \mathrm{h}$. PN was discontinued after $48 \mathrm{~h}$ and thereafter only enteral (luminal) nutrition (EN) was provided every $3 \mathrm{~h}$ at $15 \mathrm{~mL} / \mathrm{kg}$ via an orogastric catheter. Pigs from the first litter were randomly assigned to receive bovine colostrum $(-\mathrm{C} ; n=$ 5 ) or a formula $(-\mathrm{F} ; n=14)$ that provided a similar intake of calories and protein (14). Based on previous studies, NEC lesions were expected to develop within $36 \mathrm{~h}$ after starting $\mathrm{EN}$ in $20-40 \%$ of $-\mathrm{C}$ and up to $80 \%$ of the $-\mathrm{F}$ pigs.

Pigs from the remaining three litters were assigned to four groups to examine if minimal enteral nutrition (MEN) during PN protects against NEC. The pigs received $3 \mathrm{~mL} / \mathrm{kg}$ of MEN every $3 \mathrm{~h}$ during $\mathrm{PN}$, which represented $20 \%$ of the total calories provided and is comparable to the MEN administered to very low birth weight infants $(20 \mathrm{~mL} / \mathrm{kg} / \mathrm{d} ; 17)$ and to infants recovering from NEC (18). The first group (CC; $n=10$ ) received colostrum during and after the period of PN. The second group (CF; $n=12)$ received colostrum during and formula after PN. The third group received formula during and after PN (FF; $n=12$ ). The fourth group (SF; $n=12$ ) was given an identical volume of $0.9 \%$ saline during PN and formula after the period of PN, and was expected to develop a high incidence and severity of NEC.

Pigs were killed (pentobarbitone; $60 \mathrm{mg} / \mathrm{kg}$, IV) for collection and evaluation of tissues when NEC was observed (abdominal distension, lethargy, bloody diarrhea) or after $36 \mathrm{~h}$ of EN. The entire GIT was rapidly removed and the dimensions (length and weight) of the stomach, small intestine, and colon were recorded. Each of five regions (stomach, proximal, middle, and distal small intestine, and colon) was visually examined by a minimum of two independent and experienced individuals and assigned a NEC score (14). A pig was considered to have NEC if the average score for the five regions exceeded 1.4 or a score of 3 was recorded in at least one region.

Abbreviations: EN, enteral nutrition; GIT, gastrointestinal tract; GLUT2, facilitative glucose transporter 2; MEN, minimal enteral nutrition; NAD, nicotinamide adenine dinucleotide; NEC, necrotizing enterocolitis; PN, parenteral nutrition; SGLT-1, sodium-glucose cotransporter 1 
In vivo measurement of $\boldsymbol{D}$-galactose absorption. Each pig received 15 $\mathrm{mL} / \mathrm{kg}$ of $0.9 \%$ saline with galactose and mannitol $(50$ and $20 \mathrm{~g} / \mathrm{L}$, respectively) via the orogastric catheter at conclusion of PN and after $30 \mathrm{~h}$ of EN Pigs that developed clinical signs of NEC before the second bolus were killed and necropsied. Arterial blood samples were collected before and at 20, 40, and $60 \mathrm{~min}$ after the galactose/mannitol/saline bolus was administered. The plasma was separated, deproteinized using perchloric acid, and analyzed for concentrations of mannitol by a modification of a method based on reduction of nicotinamide adenine dinucleotide (NAD) using mannitol dehydrogenase (19) and for galactose using a kit based on galactose-dehydrogenase (Boehringer Mannheim, Darmstadt, Germany).

In vitro measurement of $\boldsymbol{D}$-glucose uptake. Intact tissue glucose uptake was measured (14) for all pigs using segments of mid jejunum without focal points of NEC, unless the entire segment was afflicted. The tissues were incubated in Ringers with tracer concentrations of ${ }^{14} \mathrm{C}$ D-glucose $(0.004 \mathrm{mM})$ and ${ }^{3} \mathrm{H}$ L-glucose, and with either $25 \mathrm{mM}$ adenosine or mannitol (control) to determine whether carrier-mediated glucose uptake could be stimulated (20). Additional tissues were prepared from pigs in the third and fourth litters to measure tracer D-glucose uptake in the presence of $0.5 \mathrm{mM}$ phloridzin (PZ) or phloretin to inhibit sodium-dependent glucose transporter (SGLT1) and the facilitative diffusion glucose transporter (GLUT2), respectively. The use of tracer concentration of D-glucose provides a sensitive indicator of the activities of the apical membrane glucose transporters, and particularly by the high affinity SGLT-1 and provides greater sensitivity for detecting differences in carrier-mediated glucose uptake among pigs with and without NEC.

Statistical analysis. Data were analyzed using SAS (Version 8.2; Cary, $\mathrm{NC}$ ) and values in figures and text are means and standard error of the mean. A repeated measures approach was incorporated in the PROC MIXED model to analyze the galactose and mannitol data. Rates of intact tissue glucose uptake were analyzed by the PROC GLM procedure to search for the effects of treatment and litter, and by Duncan's test to identify specific differences. Due to the presence of a litter effect, litter was included in the analysis of treatment as a random variable. For all comparisons, $p<0.05$ was accepted as the critical level of significance.

\section{RESULTS}

Incidence and severity of NEC. All pigs fed colostrum completed the study. Due to clinical symptoms of NEC, $20-40 \%$ of the pigs in the other groups were killed before conclusion of $36 \mathrm{~h}$ of feeding. Pigs fed formula after PN without MEN (-F and SF) had the highest regional and average NEC scores (Fig. 1), with NEC lesions observed in at least one region, and $87 \%$ (20 of 23) had NEC scores greater than 1.4. Clinical symptoms of NEC appeared rapidly with

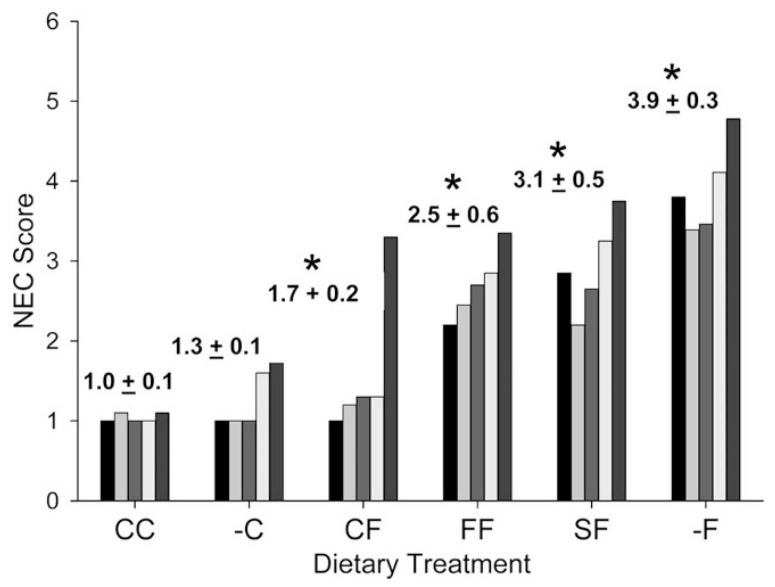

Figure 1. Average NEC scores in the stomach, proximal, mid, and distal SI, and colon (bars 1-5, respectively) of pigs receiving only PN before conversion to EN with colostrum (-C) or formula (-F), or receiving MEN as colostrum before transfer to $\mathrm{EN}$ with formula or colostrum (CF and $\mathrm{CC}$, respectively), or receiving formula during and after PN (FF). Values above each set of bars are average NEC scores \pm SEM for all five regions. Asterisks indicate average NEC score for the entire GIT is higher than value for CC pigs. some pigs experiencing bloating, diarrhea, and lethargy within $6 \mathrm{~h}$ after starting EN. Providing formula during and after PN (FF) resulted in a lower incidence and severity of NEC (64\%; 7 of 11 with scores greater than 1.4). Providing colostrum during $\mathrm{PN}$ and formula after $(\mathrm{CF})$ caused a further reduction in NEC incidence $(55 \% ; 6$ of 11) and severity in all regions, except in the colon. Feeding colostrum to pigs after, but not during PN (-C group) did not prevent NEC lesions (33\% incidence), but the severity in the different regions was lower than in CF pigs. Only one of the 10 pigs fed colostrum during and after PN (CC) had NEC. This was restricted to the colon and the average NEC score for this pig (1.2) was not considered indicative of NEC.

In vivo absorption of D-galactose and mannitol. Galactose was not detected in plasma before administering the bolus. Plasma galactose concentrations were highest $20 \mathrm{~min}$ after the bolus and declined thereafter (Fig. 2, top panel). Galactose concentrations analyzed across the samples, collected at 20, 40 , and 60 min during PN, were lower $(p<0.05)$ for FF pigs compared with $\mathrm{CC}$ and $\mathrm{CF}$ littermates (pooled data) and tended to be lower compared with SF pigs $(p=0.06)$. CC and CF pigs did not have higher galactose concentrations relative to SF pigs ( $p>0.5$ for comparison of PN with pooled data for $\mathrm{CC}$ and $\mathrm{CF}$ pigs). Plasma mannitol concentrations during PN were relatively consistent at 20,40, and $60 \mathrm{~min}$ and were higher for $\mathrm{CC}$ and $\mathrm{CF}$ pigs compared with FF (Fig. 3, top panel; $p<0.001)$ and SF $(p<0.05)$ pigs.

Plasma galactose concentrations during EN remained higher for CC pigs and were comparable with concentrations

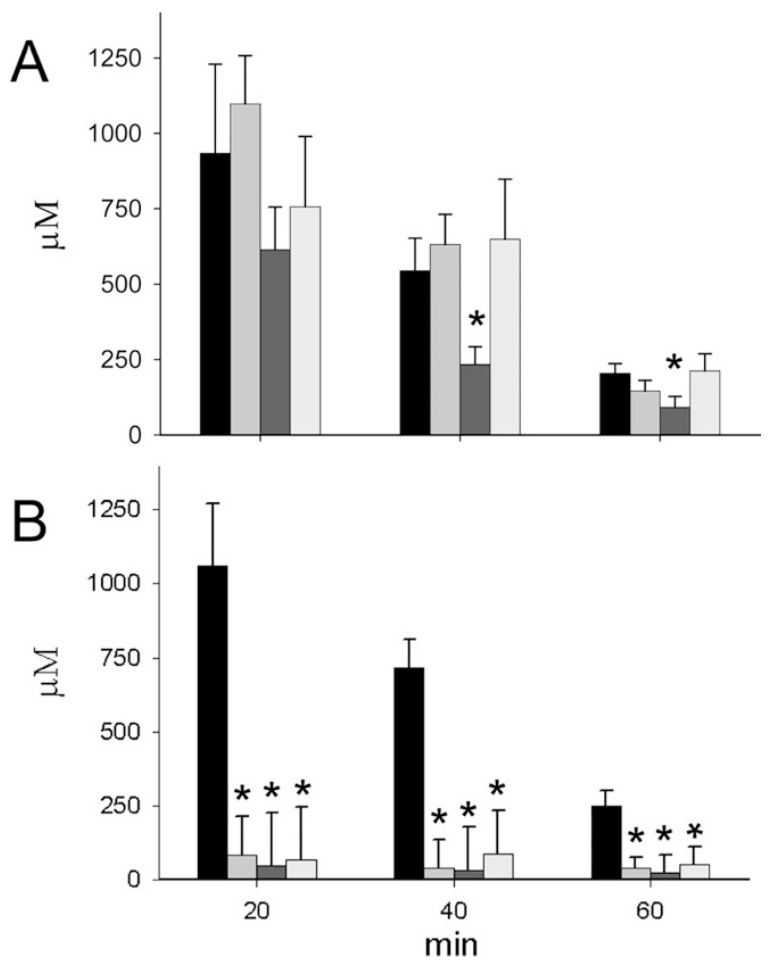

Figure 2. Plasma galactose concentrations (means \pm SEM) measured during PN (A) and EN (B) after giving an oral dose to pigs receiving MEN as colostrum during PN and subsequently EN as colostrum and formula (CC and $\mathrm{CF}$, bars 1 and 2, respectively), or formula during and after PN (FF, bar 3), or saline during PN and formula for EN (SF, bar 4). Asterisks above bars indicate values are significantly lower $(p<0.05)$ compared with $\mathrm{CC}$ pigs. 

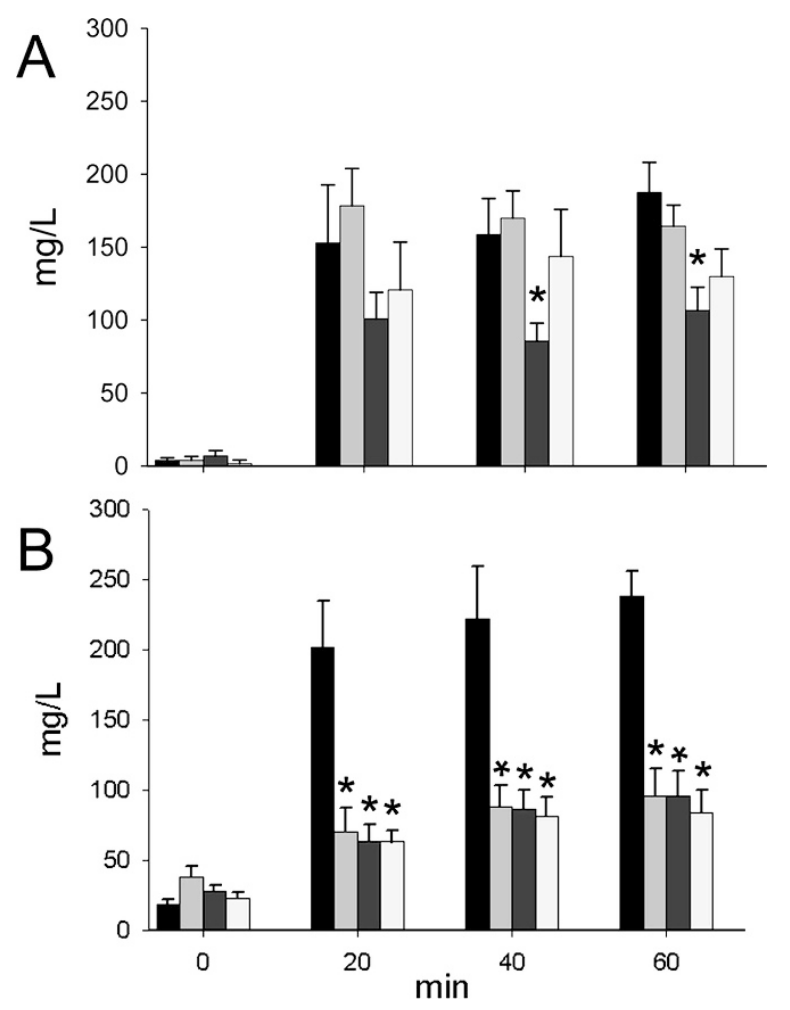

Figure 3. Plasma mannitol concentrations (means \pm SEM) measured during PN $(A)$ and EN $(B)$ after giving an oral dose to pigs receiving MEN as colostrum during PN and subsequently EN as colostrum and formula (CC and $\mathrm{CF}$, bars 1 and 2, respectively), or formula during and after PN (FF, bar 3), or saline during PN and formula for EN (SF, bar 4). Asterisks above bars indicate values are significantly lower $(p<0.05)$ compared with CC pigs.

measured in the same pigs during PN (Fig. 2, bottom panel). Values were lower $(p<0.0001)$ for pigs fed formula, regardless of diet during PN (Fig. 3, bottom panel; $p<0.001$ for comparison of $\mathrm{CC}$ and $\mathrm{CF}$ pigs). Plasma mannitol concentrations for $\mathrm{CC}$ pigs were higher during $\mathrm{EN}(p<0.05)$, whereas concentrations declined in the remaining groups that received formula.

Galactose concentrations during EN were related to NEC score, and at $20 \mathrm{~min}$ were higher for pigs with NEC scores of 1 , lowest for those with scores $>3$, with intermediate values measured in pigs with NEC scores of 1.2-1.4 and 1.5-3 (Fig. 4). Interestingly, the two pigs with NEC scores of 1 and low plasma galactose concentrations at $20 \mathrm{~min}$ were $\mathrm{CF}$ pigs, whereas the remaining pigs with NEC scores of 1 were CC pigs. Furthermore, the single CC pig with evidence of NEC (average score of 1.2) had a plasma galactose concentration of $540 \mu \mathrm{M}$ at $20 \mathrm{~min}$, whereas the remaining $\mathrm{CC}$ pigs had an average concentration of $1251 \pm 263 \mu \mathrm{M}$. These findings suggest the two CF pigs with NEC scores of 1 may have been at increased risk of NEC, but had not yet developed symptoms or obvious mucosal pathology. Mannitol concentrations were also highest in CC pigs with NEC scores of 1 and lower in pigs with any sign of NEC anywhere in the GIT (data not shown).

In vitro uptake of D-glucose. Due to a significant litter effect, comparisons among treatments for glucose uptake by the intact tissues were made after expressing values for each

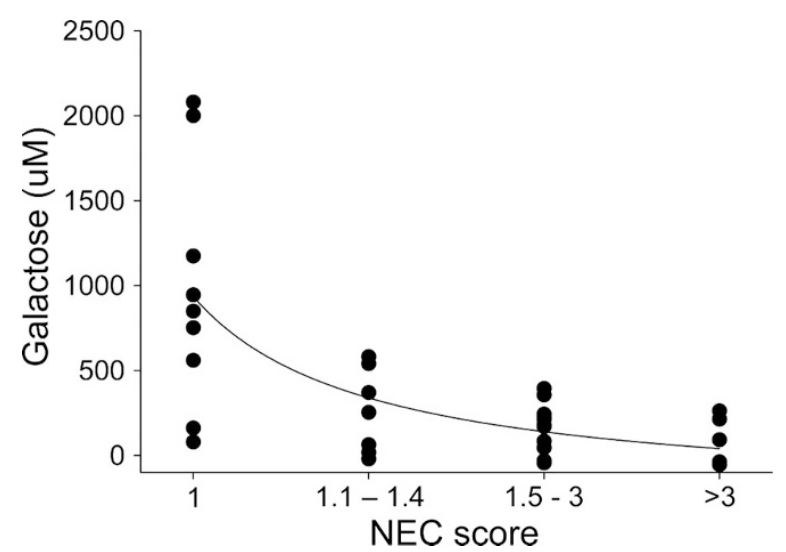

Figure 4. Plasma galactose concentrations measured $20 \mathrm{~min}$ after administration of an oral bolus to individual pigs with different magnitudes of NEC and receiving EN as colostrum or formula. NEC scores were assigned at necropsy and are the average for the five regions of the gastrointestinal tract. NEC scores were grouped as no evidence of NEC (1), mild, nonclinical NEC (1.1-1.4), moderate NEC (1.5-3), and severe NEC $(>3)$.

pig as a percentage of glucose accumulation by $-\mathrm{C}$ or $\mathrm{CC}$ littermates without NEC and considered as healthy controls. These comparisons revealed that rates of glucose accumulation by SF pigs were $36 \pm 26 \%$ of values measured in healthy CC littermates $(p<0.05)$. Similarly, rates of glucose uptake by -F pigs averaged $<20 \%$ of uptakes for -C littermates. Rates of glucose uptake for the $\mathrm{CF}$ and FF pigs averaged $42 \pm 24 \%$ and $55 \pm 35 \%$ of $\mathrm{CC}$ littermates, but due to individual variation the comparisons with $\mathrm{CC}$ littermates were not significant $(0.10>p>0.05)$.

There was a significant linear relationship $(r=0.75 ; p<$ 0.01 ) between glucose uptake by intact tissues and the severity of NEC averaged over the entire GIT. Specifically, the highest rates of glucose uptake were measured from animals with average NEC scores of 1 (Fig. 5). Glucose uptake was nearly $40 \%$ lower for pigs with low NEC scores (1.1-1.4). In most of these pigs, the NEC was restricted to the colon and the small intestine was assigned a score of 1 and considered "healthy." The lowest rates of uptake were for pigs with high NEC scores $(>3)$, and with involvement of the small intestine. Obviously, the presence of NEC in any region of the GIT compromises the ability of the small intestine to absorb glucose, and particularly when NEC lesions are in the small intestine.

PZ inhibited SGLT-1 mediated glucose uptake by $>90 \%$ when the tissues were from pigs with NEC scores between 1 and 1.5 (Fig. 6). PZ decreased the already lower glucose uptake by less than $30 \%$ when the tissues were from pigs with severe NEC (scores $>3$ ). The magnitude of glucose uptake inhibition by $\mathrm{PZ}$ was highly variable for tissues from pigs with intermediate NEC scores (1.5-3). Exposing tissues to phloretin did not decrease glucose uptake relative to unexposed tissues (data not presented).

When tissues from pigs with NEC scores of 1 were exposed to $25 \mathrm{mM}$ adenosine, glucose uptake increased $29 \%$ compared with adjacent tissues exposed to mannitol (data not presented). In contrast, glucose uptake by tissues from pigs with NEC scores of 3 or greater did not increase in response to adenosine 


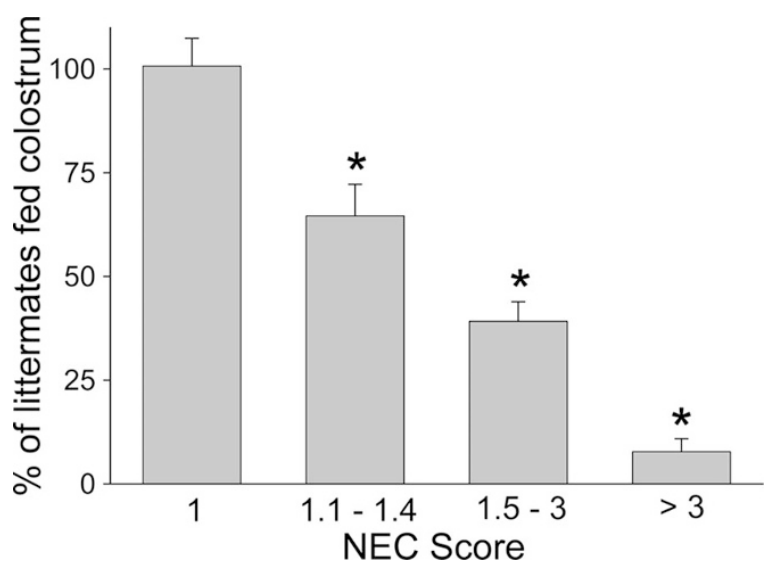

Figure 5. Rates of glucose uptake (means \pm SEM) by intact tissues harvested from pigs with no evidence of NEC (1), with mild, nonclinical NEC (1.1-1.4), moderate NEC (1.5-3), and severe NEC $(>3)$. Asterisks above bars indicate values are significantly lower $(p<0.05)$ compared with littermates fed colostrum.

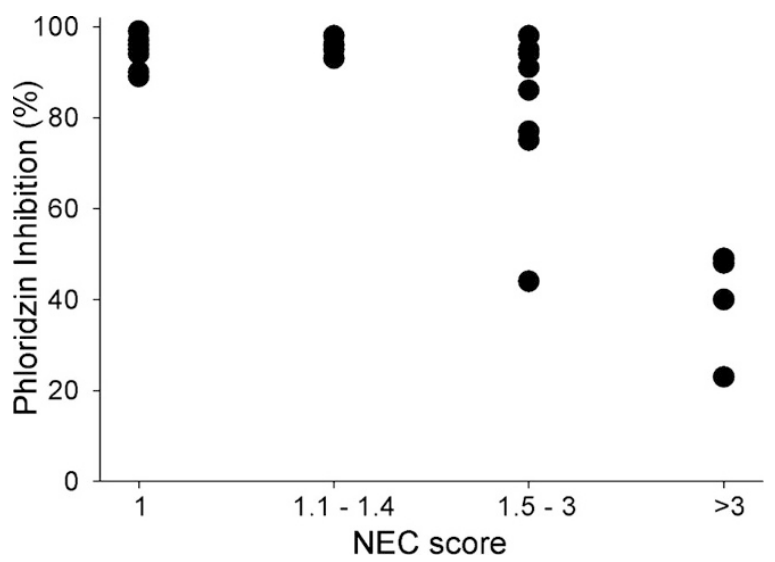

Figure 6. The relationship between severity of NEC lesions and the percentage of glucose uptake by intact tissues that is inhibited by phloridzin.

exposure, with intermediate responses for tissues from pigs with NEC scores of 1.5-3.

\section{DISCUSSION}

NEC remains a leading cause of morbidity and mortality for preterm infants, despite intense efforts to reduce the incidence and severity. Although the proximate causes for the focal points of ischemia and necrosis remain uncertain (21), the present results confirm our previous findings (14) that composition of enteral feeds plays an important role in determining the risk of NEC. Importantly, our findings indicate the development of even mild NEC in the distal bowel (colon) decreases the capacities of an apparently healthy small intestine to absorb nutrients.

The last $10 \%$ of gestation for pigs is important for intestinal growth and development of carrier-mediated absorption of nutrients (22). Due to fear of NEC, preterm infants are often placed on PN, which reduces intestinal growth and delays development of functions of newborn pigs, including nutrient absorption (23). Although none of the pigs developed clinical signs of NEC during the period of PN, the present findings indicate the source of MEN is an important determinant of
GIT characteristics. During PN, the absorption of mannitol, which is independent of transporters, was higher $(p<0.05$ all time points were pooled) when pigs received colostrum as the source of MEN (CC and $\mathrm{CF}$ ) compared with those receiving saline (SF) or formula (FF). However, in vivo uptake of galactose, which is absorbed by carrier-mediated and carrierindependent diffusion, was not increased during PN by colostrum relative to saline, whereas the galactose absorption by FF pigs was lower $(p<0.05)$. Even though intestinal characteristics were not directly measured, the results suggest using colostrum for MEN during PN increases passive diffusion, but this apparently does not increase the number of transporters, whereas formula decreases both passive diffusion and carrier-mediated transport.

The adverse influence of the formula on intestinal characteristics was even more pronounced after the transition to EN. The lower galactose values before necropsy for the CF pigs demonstrate that providing colostrum during PN does not fully protect against NEC when infants are fed after PN an enteral formula that increases the risk of NEC. Another consideration is that all surviving pigs were examined $36-40 \mathrm{~h}$ after the start of EN. It is possible that the incidence and severity of NEC scores would have been higher if the time of formula feeding had been extended.

The ability to identify preterm infants at risk of NEC has remained elusive (24), and accelerating the transition to EN increases the risk (17). After the conversion to EN, the lower galactose concentrations measured in all but the CC pigs suggest a decrease in aldohexose absorption and is indicative of an increased risk of NEC (10). At the time of necropsy, many of the pigs with NEC scores between 1.2 and 3 did not exhibit overt signs of NEC (e.g., bloating, diarrhea). Yet, just before the necropsy the same pigs had plasma galactose concentrations at $20 \mathrm{~min}$ that were lower than $600 \mu \mathrm{M}$. This includes pigs with NEC restricted to the colon and those with NEC scores between 1.2 and 1.4, which is not considered as indicative of NEC. In contrast, plasma galactose concentrations averaged two-fold higher for $\mathrm{CC}$ pigs without evidence of NEC in any region. These findings indicate plasma concentrations after an oral dose of galactose and perhaps other solutes absorbed by carrier-mediated pathways may be clinical indicators of the early onset or presence of NEC, despite the absence of overt clinical symptoms.

The present results document the negative impact of NEC, regardless of severity and location, on the abilities of the small intestine to absorb nutrients. The lower rates of glucose uptake by intact small intestinal tissue from pigs with NEC are consistent with malabsorption and are corroborated by lower plasma galactose concentrations after an oral bolus. Moreover, the PZ data indicate that the majority of glucose uptake by tissues from pigs considered healthy or with mild NEC was via SGLT-1, that NEC causes a decline in glucose uptake by SGLT-1, presumably by reducing the number of functioning transporters. The lack of a decline in glucose uptake by tissues exposed to phloretin implies that GLUT2 plays a minimal role in glucose uptake during this stage of development and when NEC is present. However, the tracer concentrations of glucose used in the uptake solution may not have been high enough to 
drive glucose uptake by facilitative transporters, such as GLUT2. Therefore, the role of GLUT2 in absorption may have been underestimated.

The decreased or absent response to adenosine by the small intestine of pigs with scores greater than 1 indicates NEC compromises the ability of the intestine to respond to stimuli that rapidly up-regulate the absorption of glucose and probably other nutrients. This would exacerbate the already reduced carrier-mediated capacities to absorb nutrients. It is unknown if NEC disrupts the adenosine receptors, the signaling pathways, or the mechanisms that are responsible for the rapid increase in absorptive functions.

Paracellular absorption has been proposed as a major mechanism of nutrient absorption (25). Yet, paracellular absorption does not seem to play an important role in premature pigs, particularly those with NEC. Specifically, despite a healthy small intestine based on macroscopic clinical observations, pigs that had NEC restricted to the colon had reduced plasma galactose concentrations. This reduction occurred despite an oral galactose challenge that was sufficiently high to trigger possible changes in tight junctions and open the paracellular pathway of absorption. Moreover, other studies have shown that NEC increases plasma concentrations of inflammatory cytokines, such as tumor necrosis factor- $\alpha$ (TNF- $\alpha$ ), Interleukin (IL)-1 $\beta$, IL-6, and IL-8 (24,26,27), which disrupt tight junctions $(28,29)$, are associated with increased paracellular permeability (30), lead to failure of the gut barrier (26), and coincide with increased mucosal permeability to sugars (31). Collectively, these findings lead to the prediction that NEC should increase paracellular movement of solutes, including galactose. Yet, plasma galactose concentrations were lower in pigs with NEC, corresponding with the decline in carriermediated glucose uptake by intact tissues. Apparently, nutrient absorption by neonates is more dependent on carriermediated pathways, and these are disrupted by NEC.

The signals that trigger the decline in carrier-mediated nutrient absorption by the healthy small intestine, despite NEC being restricted to the colon, remain unknown. The most likely candidates are the elevated plasma concentrations of inflammatory cytokines and bacterial endotoxins that are associated with NEC. Exemplary is the decreased sugar absorption by intestinal tissue exposed to TNF- $\alpha(32,33)$. It is unknown if therapeutic regimens that reduce inflammatory cytokines and the associated mucosal responses of infants with NEC (34-37) will improve nutrient absorption. The lower incidence and severity of NEC in the CC pigs may be related to colostrum reducing the secretion of inflammatory cytokines by gut epithelial cells and decreasing bacterial adherence (38). The present study did not include pigs given formula during PN and converted to colostrum for EN, as this was not considered a clinically relevant treatment. Therefore, it is not possible to determine whether colostrum can compensate for the adverse impact associated with providing the formula during PN. However, the reduced incidence and severity of NEC lesions among -C pigs indicate that providing colostrum as the source of enteral nutrients does confer protection.

\section{REFERENCES}

1. Kafetzis DA, Skevaki C, Costalos C 2003 Neonatal necrotizing enterocolitis: an overview. Curr Opin Infect Dis 16:349-355

2. Luig M, Lui K; NSW and ACT NICUS Group 2005 Epidemiology of necrotizing enterocolitis-Part I: Changing regional trends in extremely preterm infants over 14 years. J Paediatr Child Health 41:169-173

3. Berseth CL 2005 Feeding strategies and necrotizing enterocolitis. Curr Opin Pediatr $17: 170-173$

4. Lin PW, Stoll BJ 2006 Necrotising enterocolitis. Lancet 368:1271-1283

5. Stoelhorst GM, Rijken M, Martens SE, Brand R, den Ouden AL, Wit JM, Veen S; Leiden Follow-Up Project on Prematurity 2005 Changes in neonatology: comparison of two cohorts of very preterm infants (gestational age $<32$ weeks): the Project On Preterm and Small for Gestational Age Infants 1983 and the Leiden Follow-Up Project on Prematurity 1996-1997. Pediatrics 115:396-405

6. Dai D, Walker WA 1999 Protective nutrients and bacterial colonization in the immature human gut. Adv Pediatr 46:353-382

7. Bjornvad CR, Schmidt M, Petersen YM, Jensen SK, Offenberg H, Elnif J, Sangild PT 2005 Preterm birth makes the immature intestine sensitive to feeding-induced intestinal atrophy. Am J Physiol Regul Integr Comp Physiol 289:R1212-R1222

8. Cheu HW, Brown DR, Rowe MI 1989 Breath hydrogen excretion as a screening test for the early diagnosis of necrotizing enterocolitis. Am J Dis Child 143:156-159

9. Garstin WI, Boston VE 1987 Sequential assay of expired breath hydrogen as a means of predicting necrotizing enterocolitis in susceptible infants. J Pediatr Surg 22:208210

10. Shulman RJ, Wong WW, Smith EO 2005 Influence of changes in lactase activity and small-intestinal mucosal growth on lactose digestion and absorption in preterm infants. Am J Clin Nutr 81:472-479

11. Gibbs K, Lin J, Holzman IR 2007 Necrotising enterocolitis: the state of the science. Indian J Pediatr 74:67-72

12. Sigalet DL, Lees GM, Aherne FX, Fedorak R, Keelan M, Thomson AB, van Aerde J 2001 Nutritional effects of surgical and medical treatment for short bowel syndrome. JPEN J Parenter Enteral Nutr 25:330-336

13. Liefaard G, Heineman E, Molenaar JC, Tibboel D 1995 Prospective evaluation of the absorptive capacity of the bowel after major and minor resections in the neonate. J Pediatr Surg 30:388-391

14. Sangild PT, Siggers RH, Schmidt M, Elnif J, Bjornvad CR, Thymann T, Grondah ML, Hansen AK, Jensen SK, Boye M, Moelbak L, Buddington RK, Westrom BR, Holst JJ, Burrin DG 2006 Diet- and colonization-dependent intestinal dysfunction predisposes to necrotizing enterocolitis in preterm pigs. Gastroenterology 130:17761792

15. Sangild PT, Schmidt M, Elnif J, Bjornvad CR, Westrom BR, Buddington RK 2002 Prenatal development of gastrointestinal function in the pig and the effects of fetal esophageal obstruction. Pediatr Res 52:416-424

16. Oste M, Van Ginneken CJ, Van Haver ER, Bjornvad CR, Thymann T, Sangild PT 2005 The intestinal trophic response to enteral food is reduced in parenterally fed preterm pigs and is associated with more nitrergic neurons. J Nutr 135:2657-2663

17. Berseth CL, Bisquera JA, Paje VU 2003 Prolonging small feeding volumes early in life decreases the incidence of necrotizing enterocolitis in very low birth weight infants. Pediatrics 111:529-534

18. Bohnhorst B, Muller S, Dordelmann M, Peter CS, Petersen C, Poets CF 2003 Early feeding after necrotizing enterocolitis in preterm infants. J Pediatr 143:484-487

19. Graefe H, Gütschow B, Gehring H, Dibbelt L 2003 Sensitive and specific photometric determination of mannitol in human serum. Clin Chem Lab Med 41:10491055

20. Kimura Y, Turner JR, Braasch DA, Buddington RK 2005 Lumenal adenosine and AMP rapidly increase glucose transport by intact small intestine. Am J Physiol Gastrointest Liver Physiol 289:G1007-G1014

21. Lee JS, Polin RA 2003 Treatment and prevention of necrotizing enterocolitis. Semin Neonatol 8:449-459

22. Buddington RK, Malo C 1996 Intestinal brush-border membrane enzyme activities and transport functions during prenatal development of pigs. J Pediatr Gastroenterol Nutr 23:51-64

23. Sangild PT, Tappenden KA, Malo C, Petersen YM, Elnif J, Bartholome AL, Buddington RK 2006 Glucagon-like peptide 2 stimulates intestinal nutrient absorption in parenterally fed newborn pigs. J Pediatr Gastroenterol Nutr 43:160-167

24. Upperman JS, Camerini V, Lugo B, Yotov I, Sullivan J, Rubin J, Clermont G, Zamora R, Ermentrout GB, Ford HR, Vodovotz Y 2007 Mathematical modeling in necrotizing enterocolitis-a new look at an ongoing problem. J Pediatr Surg 42:445-453

25. Pappenheimer JR 1998 Scaling of dimensions of small intestines in non-ruminant eutherian mammals and its significance for absorptive mechanisms. Comp Biochem Physiol A Mol Integr Physiol 121:45-58

26. Sharma R, Tepas JJ III, Hudak ML, Mollitt DL, Wludyka PS, Teng RJ, Premachandra BR 2007 Neonatal gut barrier and multiple organ failure: role of endotoxin and proinflammatory cytokines in sepsis and necrotizing enterocolitis. J Pediatr Surg 42:454-461

27. Noerr B 2003 Current controversies in the understanding of necrotizing enterocolitis. Part 1. Adv Neonatal Care 3:107-120

28. Wang F, Schwarz BT, Graham WV, Wang Y, Su L, Clayburgh DR, Abraham C, Turner JR 2006 IFN-gamma-induced TNFR2 expression is required for TNFdependent intestinal epithelial barrier dysfunction. Gastroenterology 131:1153-1163

29. Utech M, Bruwer M, Nusrat A 2006 Tight junctions and cell-cell interactions. Methods Mol Biol 341:185-195 
30. Prasad S, Mingrino R, Kaukinen K, Hayes KL, Powell RM, MacDonald TT, Collins JE 2005 Inflammatory processes have differential effects on claudins 2, 3 and 4 in colonic epithelial cells. Lab Invest 85:1139-1162

31. Dupont C, Goutail-Flaud MF 1990 Alterations of intestinal permeability to sugars in infants following neonatal surgery. J Pediatr Gastroenterol Nutr 11:66-71

32. Amador P, Garcia-Herrera J, Marca MC, de la Osada J, Acin S, Navarro MA, Salvador MT, Lostao MP, Rodriguez-Yoldi MJ 2007 Inhibitory effect of TNF-alpha on the intestinal absorption of galactose. J Cell Biochem 101:99-111

33. Garcia-Herrera J, Navarro MA, Marca MC, de la Osada J, Rodriguez-Yoldi MJ 2004 The effect of tumor necrosis factor-alpha on D-fructose intestinal transport in rabbits. Cytokine 25:21-30

34. Travadi J, Patole S, Charles A, Dvorak B, Doherty D, Simmer K 2006 Pentoxifylline reduces the incidence and severity of necrotizing enterocolitis in a neonatal rat model. Pediatr Res 60:185-189
35. Halpern MD, Clark JA, Saunders TA, Doelle SM, Hosseini DM, Stagner AM, Dvorak B 2006 Reduction of experimental necrotizing enterocolitis with anti-TNFalpha. Am J Physiol Gastrointest Liver Physiol 290:G757-G764

36. Zhang BH, Yu HG, Sheng ZX, Luo HS, Yu JP 2003 The therapeutic effect of recombinant human trefoil factor 3 on hypoxia-induced necrotizing enterocolitis in immature rat. Regul Pept 116:53-60

37. Hsueh W, Caplan MS, Qu XW, Tan XD, De Plaen IG, Gonzalez-Crussi F 2003 Neonatal necrotizing enterocolitis: clinical considerations and pathogenetic concepts. Pediatr Dev Pathol 6:6-23

38. Brooks HJ, McConnell MA, Corbett J, Buchan GS, Fitzpatrick CE, Broadbent RS 2006 Potential prophylactic value of bovine colostrum in necrotizing enterocolitis in neonates: an in vitro study on bacterial attachment, antibody levels and cytokine production. FEMS Immunol Med Microbiol 48:347-354 